\title{
GENÈSE ET FACTICITÉ
}

\author{
FLORIAN FORESTIER
}

\begin{abstract}
By opening the investigation to scattered and inchoative states, Marc Richir's phenomenology reverses the relation between legitimation and receptivity. He calls into question the idea of a spontaneous adhesion of the experience to itself and reformulates the Husserlian problematic of Ur-doxa without abandoning the ambition of legitimation. Legitimation is no longer ensured by the donation of a sense but by a gesture of reinstitution.
\end{abstract}

La phénoménologie de Marc Richir renverse la relation de la légitimation à la réceptivité :

- D'une part, il ne fait plus de la donation de la chose en chair et en os et de l'ouverture à l'horizon du monde comme totalité un paradigme, et concentre ses analyses sur les expériences de flottement et de dispersion, sur les états « inchoatifs » pour reprendre sa terminologie.

- D’autre part, il lie l'élucidation phénoménologique des « formations de sens » et de la connaissance à l'obscurité dont notre expérience est tissée et met ainsi en œuvre une " phénoménologie de l'inapparent ${ }^{1}$ » (selon le terme de Heidegger) originale.

Ces deux questions sont elles-mêmes liées à une décision théorique préalable considérable : rendre problématique l'adhésion spontanée de l'expérience à ellemême que Husserl thématise avec l'idée d'Ur-doxa, sans pour autant abandonner l'ambition de légitimation (c'est-à-dire d'élucidation de ce par quoi une connaissance est possible) qui traverse la phénoménologie husserlienne. Cette légitimation

1 Heidegger Martin, Séminaire de Zähringen, in Questions IV, 1976, p. 487. 
n'est plus pensée dans une optique cartésienne par une théorie de l'élucidation phénoménologique de l'évidence apodictique, mais, dans une perspective plus fichtéenne, comme processus actif et ininterrompu de réinstitution. Nous sommes en perpétuel travail de réveil, de recomposition, de rassemblement.

\section{La facticité et l'expérience}

La plus forte critique que la démarche phénoménologique doit affronter est sans doute celle qui met en question sa possibilité à la fois pratique et théorique. Cette critique, d'inspiration wittgensteinienne, et dont Jocelyn Benoist est peut-être actuellement le porteur le plus acéré ${ }^{2}$, considère en effet que la démarche phénoménologique procède d'une erreur catégorielle. Il n'y aurait pas de sens en effet à vouloir caractériser et rendre compte de l'expérience en général, car on ne peut, par définition, se placer à l'extérieur de celle-ci et trouver un élément de comparaison par rapport auquel celle caractérisation aurait un contenu. Toute prise de position sur l'expérience étant nécessairement partie-prenante de celle-ci, les concepts utilisés par la phénoménologie ne peuvent être que vides. Arrachés aux contextes et circonstances dans lesquelles leur usage a du sens et à la mesure desquels ils ont été forgés, ils ne constituent rien de plus que des effets de discours enrôlés dans des spéculations idéalistes et dogmatiques.

A.

La meilleure réponse à apporter à cette objection est transcendantale. Elle conduit à faire de cette apparente contradiction la racine de la phénoménologie, qui ne peut être réellement mise en ouvre qu'à condition d'exposer d'abord la nature et les termes du "paradoxe». Cette orientation n'est certes pas sans conséquences sur la nature de la phénoménologie, car elle revient à remettre en question le rôle et même la possibilité d'une description phénoménologique tels que les phénoménologues l'entendent le plus souvent. Dans notre optique en effet, la phénoménologie est liée à un geste spéculatif préalable, lequel conditionne la possibilité des descriptions et leur sens. Ce geste spéculatif revient à poser que l'expérience n'est pas « simple ", qu'elle ne coïncide jamais avec son actualité, que nous ne sommes pas pris sans reste dans ce que nous vivons ou ce qui nous arrive.

2 Benoist Jocelyn, Eléments de philosophie réaliste, Paris, Vrin, 2010. 
Le concept le plus pertinent que nous lègue la tradition pour rendre compte de cet aspect est celui de " réflexibilité ${ }^{3}$ " que Fichte met en exergue dans la Wissenschaftslehre de 1813 : aucun contenu d'expérience, quel qu'il soit, ne s'impose absolument au moi qui se définit comme réflexivité potentielle, puissance de distanciation latente. L'attestation première, «indirecte ${ }^{4}$ ", de cette " réflexivité phénoménologique » est le fait que nous puissions parler de l'expérience comme expérience, la comprendre comme nôtre.

Nous existons en nous différenciant de notre exister et ne sommes tout d'abord jamais irréductiblement absorbés dans nos actions, nos engagements. Même totalement engagés dans une action, par exemple lorsque nous gravissons une paroi rocheuse, nous sommes affectés, même passagèrement par toutes sortes d'autres choses (une fleur, une couleur, une sensation) qui ne nous traversent pas pour s'abimer dans un lac sans fond d'oubli, et que nous pouvons être amenés à réactiver pour les inscrire dans un flux temporel. Tout aussi bien, au sein même de notre action, il y a des latences, des blancs. En quelque sorte, nous ne cessons d'échapper à notre situation, aussi bien par l'intérieur (par ces latences, résistances, perturbations, dont la situation se fait, mais qui peuvent aller jusqu'à la faire éclairer) que par l'extérieur (en remarquant précisément que nous sommes en situation, et par là, en nous en distinguant).

Encore une fois, le fond de l'énigme n'est plus tant que l'expérience soit multiple et diverse, ni-même habitée d'une épaisseur, que le fait que celle-ci se manifeste, fut-ce indirectement, en elle, comme latence ou virtualité. L'expérience est en quelque sorte toujours marquée du sceau de sa facticité - elle n'est précisément jamais tout à fait «normale » (si ce terme a sens), mais toujours donnée avec, sinon la conscience, du moins le sens plus ou moins marqué de sa propre contingence. Selon la terminologie de Marc Richir, les configurations que prend l'expérience sont elles-mêmes le produit d'une genèse au sein d'un schématisme ; elles ne recouvrent pas la totalité de l'expérience dont la masse les excède toujours, et demeurent toujours provisoires, quelle que soit leur stabilité5.

3 Cf. Schnell Alexander, « Possibilité, possibilisation et réflexion de la réflexion. L'héritage de la philosophie allemande classique dans la phénoménologie transcendantale ", in Philosophiques, 43, $\mathrm{n}^{\circ}$ 2, automne 2016, pp. 297-315.

4 Abondamment utilisé par Marc Richir (cf. par exemple Phénoménologie en esquisses, Grenoble, Millon, p. 484, «le Phantasiewelt est une attestation indirecte de ce que nous nommons le champ phénoménologique sauvage ", le concept en apparence paradoxal "d'attestation indirecte » est au cœur de notre problématique. Il désigne les «traces » ou " perturbations » qui témoignent au sein d'un registre d'expérience d'un fond de processus et de virtualités latentes qui se signalent par l'intermédiaire de ces effets.

5 Cette réflexion reprend une tradition ancienne qui fait de la possibilisation et du possible la matrice de la phénoménologie. Chez Husserl, à travers la distinction fait/eidos, à travers le concept d'objet, 
B.

La question de notre origine elle-même nous rappelle la facticité des modalités selon lesquelles nous expérimentons les choses. Aussi difficile à penser que cela puisse nous sembler, la temporalité est elle-même apparue pour nous plutôt qu'avec nous : en deçà de la capacité de notre mémoire à distinguer, identifier, ordonner, nous existions déjà, et quelque chose persiste en nous de cette épaisseur chaotique et obscure de temps. Je suis né, je suis apparu, je me suis développé, il est évident que l'expérience qui a été «mienne » n’a pas immédiatement eu le caractère intentionnel, perceptuel, thétique. Pour Husserl lui-même, « il existe une possibilité originaire et génétiquement un début originaire tels qu’aucune conscience propre du temps ne soit encore constituée, c'est-à-dire que pour le moi aucun événement n'ait encore lieu en un temps. Ce serait une conscience originairement dormante, qui n'a jamais encore été vigile, ou un moi originairement dormant, qui n’a encore jamais été éveillé. ${ }^{*}$ "

Comment comprendre la genèse d'une expérience ordonnée du temps ? La phénoménologie de l'événement, telle que la développe par exemple Claude Romano, s'attache à cette question. Pour Romano, comprendre l'origine du temps nous impose de repenser ce qui « échappe par principe au phénomène de l'intra-temporalité, tel qu'il se structure à partir de l'attente, de la présentification et du souvenir ${ }^{7}$ » et « ne survient pas à proprement parler dans un monde, il ouvre un monde en survenant ${ }^{8} »$. La naissance marque ainsi une excédance absolue par rapport au vivre et instaure une expérience de " retard constitutif » du sujet (pour Romano, de l'advenant) par rapport à ses possibilités. Dans notre optique cependant, il est insuffisant de se contenter de rendre compte de la structure phénoménologique de l'événement si on ne la ramène pas aux structures du champ phénoménologique qui la rendent possible. La question essentielle est alors celle de l'articulation de l'expérience et de la facticité qui l'habite, des façons dont le virtuel infuse et parfois transforme l'actuel, de ce que Maldiney et Richir nomment «transpassibilité 9 » et « transpossibilité ${ }^{10}$ ».

donné dans son être tel, à travers l'idée d'une l'essence extractible réflexivement à travers le concept même d'intentionnalité. Chez Heidegger, à travers la réflexion sur conditions de possibilités mêmes de cette distance, de cette transcendance intentionnelle, de ce qui la sous-tend, de la thématique de la transcendance du monde. Le monde est à la fois mon monde et le monde, la forme monde dans son absoluité en mon monde, et réciproquement, mon individuation n'ayant sens que sur fond du monde.

6 Husserl Edmund, Manuscrits de Bernau sur la conscience du temps, Grenoble, Millon, 2010, p. 199.

7 Romano Claude, L'événement et le temps, Paris, Presses universitaires de France, 1998, p. 165.

8 Romano C., Il y a, Paris, Presses universitaires de France, 2003, pp. 355-356.

9 Maldiney Henri, Penser l'homme et la folie, Grenoble, Millon, 1991, p. 304.

10 Richir M., Méditations phénoménologiques, Grenoble, Millon, 1992, p. 113ss. 
Pour questionner cette autreté interne à l'expérience, il n'est pas suffisant de se limiter, comme le faisait Husserl, de quelques références à la folie, l'enfance, ou l'animalité. Si instructive soit-elle par ailleurs, la question de l'animalité risque elle-même de demeurer abstraite si la "pluralité » de l'expérience humaine n'est pas d'abord considérée pour elle-même. Il faut au contraire entrer profondément dans ces expériences que sont la fatigue, l'épuisement, la distraction, la rêverie, et bien entendu, le rêve.

Celui-ci interroge en effet profondément les concepts de temps, de l'espace, du monde. Il ouvre sur une expérience brouillée des dimensions auxquelles ceux-ci renvoient. Il constitue ainsi une forme indirecte et latente d'épochè de ces paramètres et nous en révèle une nouvelle profondeur, nous appelant à remettre en question, au-delà du seul primat de l'intentionnalité perceptive, l'idée de monde et les «structures» (de transcendance, de continuité, etc.) qui y sont traditionnellement associées.

Le rêve ne se déploie généralement pas dans un espace et un temps standards. Les catégories de l'objectivation ne semblent pas y avoir cours ; ce qui y a lieu n'est pas l'objet de visées identifiantes et réindentifiantes. Le rêve nous invite à nous intéresser à un ensemble d'états, de significativités, d'intuitions qui ne se lient pas selon la forme ordonnée d'une trame narrative spatio-temporellement déterminée : il constitue une expérience concrète mais non objectivable, qui ne peut donner prise à une analyse systématique ou eidétique classique. Chaque rêve est une expérience singulière, à laquelle la conscience n'accède que grâce à ses phases les plus proches de la veille.

C.

Le grand mérite de la phénoménologie génétique et générative ${ }^{11}$ est d'avoir su articuler la problématique du virtuel au concept fichtéen de réflexibilité pour déterminer un nouveau " lieu » à la fois théorique et concret du questionnement phénoménologique. Cette perspective nous conduit à élargir le concept de "réflexivité » au-delà de ce que nous expérimentons et de ce que nous sommes, pour en faire une donnée fondamentale de la question ontologique, de la façon dont il y a quelque chose. Selon les mots d'Alexander Schnell, la réflexivité invite la phénoménologie à poser la précarité essentielle du réel ${ }^{12}$.

11 Cf. Schnell A., La déhiscence du sens, Paris, Hermann, 2015.

12 Cf. Schnell A., «La précarité du réel. Sur le statut de la « réalité » chez J.G. Fichte et M. Richir », in Annales de Phénoménologie, $\mathrm{n}^{\circ} 11 / 2012$, pp. 93-111 ; « La contingence dans la possibilisation et dans le retrait. A propos du transcendantalisme de Heidegger ", in AUC Interpretationes, IV, no 1, 2016, pp. 129-147. 
Selon cette orientation, la réflexibilité caractérise une exigence phénoménologique et transcendantale : qu'aucune forme de la manifestation ne soit absolutisée. Elle doit se comprendre comme structure une phénoménologique originaire plutôt que comme l'activité d'un sujet négateur.

La réflexibilité ainsi constitue également une alternative aux phénoménologies de l'événement ou de l'adonnation afin de dépasser la phénoménologie intentionnelle classique. Dans sa perspective, le propre de la phénoménologie est certes de prendre la facticité au sérieux, mais cette « surprise du comment » ne veut pas dire que l'expérience soit en elle-même et toujours expérience de surprise. Il s'agit ici de faire de la facticité le thème de la phénoménologie sans pour autant l'assigner à une expérience ou une instance ni sacrifier l'existence de réflexivité et d'attestation au profit d'une passivité toujours accentuée.

Dès lors, la facticité n'est plus pensée au prisme d'une expérience particulière (de la chose ou l'événement) mais de la concrétude et de l'inchoativité de toute expérience. La légitimation pour sa part est appréhendée à partir de l'effort qui ne cesse de travailler cette expérience pour la mettre en ordre, en forme.

\section{La phénoménologie et l'imaginaire}

A.

Dès ses premières œuvres, Merleau-Ponty pense l'imaginaire comme une modalité de l'être des choses, non purement subjective, comme un concret sauvage agissant en moi. L'imaginaire n' est pas seulement pour lui un caractère du réel mais une dimension intrinsèque de la réalité, hors de laquelle aucune réalité ne peut être pensée. La réalité doit nécessairement, pour se donner comme réelle, être donné comme plastique et multiple : «voir, c'est par principe voir plus qu'on ne voit, c'est accéder à un être de latence..$^{13}$ "

Dans les derniers développements de son œuvre, Merleau-Ponty entreprend de penser l'ontologie elle-même à partir de l'imagination. Celle-ci n'est plus comprise comme modalité intentionnelle, mais sous sa forme archaïque, comme concrétude, élément, trace, esquisse : «l'objet en fil de fer dont je ne saurais dire ce qu'il est, ni combien de côtés il a etc. et qui pourtant est là ${ }^{14}$ ». Ce qui la caractérise est moins l'indétermination que le fait d'être irréductible au champ de l'objectivation : Merleau-Ponty décrit la texture de l'expérience perceptuelle comme

13 Merleau-Ponty Maurice, Signes, Paris, Gallimard, 1960, p. 29.

14 Merleau-Ponty M., Le Visible et l'Invisible, note de novembre 1960, p. 316. 
imaginaire ${ }^{15}$ parce que celle-ci ne relève pas principalement du thétique mais du " schématique ", qu'elle n'est a priori assujettie à aucun processus de donation perceptive selon la typique d'un objet prédéfini.

Merleau-Ponty n'utilise donc pas le mot imagination dans son sens usuel. Chez lui, il ne désigne pas un acte de reproduction ou de composition à partir de la perception, mais bien une autre modalité de rapport à l'être. Ce concept d'imaginaire est introduit contre une certaine conception de la perception, comprise comme perception d'un objet doté de qualités. Ce concept philosophique de perception fait en effet écran à une compréhension phénoménologique concrète du sensible. Il tend à amalgamer deux aspects conceptuellement distincts, qu'il faut justement séparer :

- D’une part, dans une perspective psychologique et génétique, les processus et aptitudes de notre système perceptif à traiter l'information ;

- D'autre part, un sens courant selon lequel percevoir est naturellement et spontanément compris comme percevoir quelque chose, comme percevoir un objet.

Cette ambiguïté conduit Husserl à considérer la perception d'un objet déterminé, doté de qualités, comme rapport primitif et premier au monde, à l'aune duquel toutes les modalités intentionnelles doivent être comprises. En conséquence, il manque la spécificité de "l'expérience du sensible » et sa variété.

On notera ici que le terme de sensible n'est pas lui-même sans poser de difficultés. Il est conceptuellement construit à partir d'une distinction avec celui de perception. Dans la tradition philosophique, le sensible est le plus souvent considéré comme la matière de la perception, mais on peut aussi, suivant Jocelyn Benoist ${ }^{16}$, distinguer les deux concepts à partir de leur usage. Dans cette perspective, le sensible n'est pas " plus primitif » que la perception, mais désigne simplement une autre dimension de l'expérience, au sein de laquelle la perception opère.

Sans se livrer à ce type d'analyse grammaticale, Merleau-Ponty n' est lui-même pas sans méfiance envers la distinction du sensible et du perceptif issue de la tradition et se détache dès l'introduction de la Phénoménologie de la Perception d'une conception empiriste faisant du sensible une simple matière pour se rallier à une lecture d'inspiration gestaltiste selon laquelle il n'y a sens à parler de sensible qu'au sein de configurations. Il parle d'ailleurs d'élémental, de brut, de sauvage, plutôt que d'élémentaire pour désigner cette texture vivante et mouvante de l'expérience, en deçà de ses formes les plus structurées, informées, symboliquement codées.

15 Dufourq Annabelle, Merleau-Ponty : une ontologie de l'imaginaire, Springer, Dordrecht, 2011 ; $f$. également les travaux d'Emmanuel de Saint Aubert.

16

Benoist J., Le bruit du sensible, Paris, Cerf, 2013. 
Encore une fois, le concept d'imaginaire est ici analogique : l'image telle que la pense Merleau-Ponty est un élément, un Wesen, non un acte structuré visant un objet comme image d'un autre.

La question de la terminologie est fondamentale pour ce type de projet phénoménologique, qui vise à contester des distinctions issues de la tradition et à appréhender des phénomènes qui semblent voués à échapper au langage ordinaire. Par principe en effet, tout concept est forgé à partir d'usages et de contextes concrets : il ne peut donc viser directement une primitivité préconceptuelle, mais seulement l'éclairer partiellement, sur fond de distinctions préalables.

Le recours au concept d'imagination en phénoménologie s'éclaire par exemple à la lumière des trois modalités d'imagination que distingue Fichte ${ }^{17}$. Ces modalités sont distinguées selon leur logique interne et le type de rapport au réel qu'elles impliquent : la simple image, l'image réfléchie, se sachant image, et l'image réfléchie au deuxième degré, imagination à proprement parler, c'est-à-dire réalisation de son objet dans l'acte de l'imaginer, imagination qui se pose en tant que posant, se comprend comme créatrice de son objet. Ces logiques internes aux trois formes d'images guident Marc Richir pour caractériser différentes modalités expérientielles : elles lui permettent en particulier de montrer que la perception telle que la conçoit Husserl constitue en réalité une expérience complexe, que sa structure rend dépendante d'autres formes d'expérience, comme nous allons le montrer maintenant.

B.

Dans sa Phénoménologie en esquisses, Marc Richir mobilise les concepts husserliens d'imagination et phantasia ${ }^{18}$ pour poursuivre, suivant l'horizon conceptuel et spéculatif qui vient d'être évoqué, le projet merleau-pontyen d'une élucidation de la texture imaginaire du réel et de la fonction des Wesens.

Cette refonte implique un réexamen des analyses de Husserl sur les différentes modalités de la conscience d'image. Husserl distingue 1) la conscience d'image proprement dite (un objet représenté par l'intermédiaire d'un autre objet, une image interne ou externe), et 2) la phantasia, visée pure et simple d'un contenu imaginaire sans représentation médiate. Si l'imagination est l'acte de représenter un objet au moins potentiellement existant, la phantasia vise, de son côté, un objet

17 Cf. pour plus de précision Schnell A., «Le statut de l'image et de l'imagination dans la phénoménologie générative ", in : Dufourq, A. (Ed.), Est-ce réel? Phénoménologies contemporaines de l'imaginaire, 2016, pp. 57-74.

18 Cf. Florian Forestier, La phénoménologie génétique de Marc Richir, Springer, 2014, le chapitre «Imagination et phantasia », pp. 83-108. 
purement fictif qui n'est pas assujetti à des critères de localisation, de cohérence, d'individuation. Tout l'enjeu pour Richir est de ne plus la considérer comme un mode dégradé ou secondaire de l'imagination, mais comme une élaboration schématique à part entière du sensible, qu'il est indispensable de comprendre pour rendre compte des autres modalités expérientielles.

Il faut encore le souligner, ces distinctions sont motivées par la structure de l'acte et non par la présence ou l'absence de référent réel ou de stimulus. La qualité des stimuli n'est sans doute pas la même lorsqu'un objet extérieur m'affecte et lorsque je le suscite, mais n'est pour autant pas pertinent de faire de la matière d'acte un critère pour distinguer les structures d'actes. Les actes de phantasia, imagination et perception doivent être distingués selon la façon dont la chose expérimentée se manifeste : la phantasia, on l'a vu, par une indiscernabilité de la forme et de la matière, l'imagination par une structure intentionnelle objectivante, la perception, enfin, par un caractère de présence et de certitude qui ne se réduit pas à l'intensité ou à la stabilité.

Ainsi, quelque chose peut être là stable devant moi sans que mon expérience de cette chose ne soit thétique. Tout aussi bien, je peux objectiver un objet présent face à moi par un acte dont la structure est celle de l'imagination. Cela se produit par exemple si je repère une chose sans la viser comme telle, ni l'insérer dans une structure de cohérence aspectuelle et causale. En d'autres termes, la perception ne se réduit pas à l'expérience d'une prégnance sensible, d'un " ancre " pour le système perceptif. Ce que nous expérimentons peut avoir une force et une solidité incontestable sans être perçu comme un objet visé dans son extériorité et son individualité.

Chez Husserl, la structure perceptive est matricielle, car elle fournit le modèle de la donation en chair et en os, donc de l'objectivité, qui permet de rendre compte de la structure des autres types d'actes intentionnels (imaginatifs, signitifs, évaluatifs, volitifs). Pour Richir, au contraire, l'élaboration intentionnelle et perceptive de l'expérience n'est pas originaire et spontanée, et ne peut donc être la racine de la compréhension de toutes les formes d'expérience. Il faut selon lui prendre pour point de départ la phantasia, et pour cela se détacher de la conception de Husserl qui assimile celle-ci à un mode dérivé de l'intentionnalité imaginative.

Le concept même de phantasia pose des difficultés au système husserlien. Il est en particulier difficile de distinguer l'appréhension de phantasia d'une aperception perceptive si on les considère l'une et l'autre comme des actes intentionnels structurés en matière et contenu d'acte. La seule solution dans ce cas semble être de faire d'une différence de matière d'acte le critère de distinction (du phantasma et de l'Empfindung) ce qui contreviendrait à la méthode phénoménologique que nous 
venons d'exposer. La solution est plutôt de remettre en cause la structure matière/ forme, qui s'avère indiscernable dans l'acte de phantasia. Lorsque je rêvasse ou phantasme « (...) ce n' est pas le phantasma qui est perçu, mais (...) l'acte tout entier (avec « son contenu » et son sens) d'imagination qui est quasi-perçu (...). ${ }^{19}$ » $\mathrm{Au}$ niveau de la phantasia, on ne peut donc pas identifier de contenu vécu au présent qui soit le corrélat d'un acte.

Richir pousse alors sa réflexion plus loin. La phantasia ne constitue pas seulement un domaine étranger à l'intentionnalité mais un domaine primitif, qu'il est nécessaire de comprendre si on veut rendre compte des actes d'imagination et de perception. Pour Richir, ce sont les moments de transpositions ${ }^{20}$ de la phantasia en actes imaginatifs qui nous invitent à accepter ce changement de point de vue : ces situations durant lesquelles je rêvasse et soudain présentifie quelque chose au sein de ma rêverie (je considère le centaure ou la fée qui ne faisaient que participer à ma rêverie). Lors de ces expériences en effet, c'est de l'intérieur de la phantasia que se forge l'acte imaginaire, par une transformation au sein de la phantasia, qui permet à l'acte d'acquérir une structure de présentification. Ces expériences sont un indice de la relation phénoménologique réelle entre phantasia, imagination et perception ${ }^{21}$.

Il faut encore noter que l'abandon de la structure intentionnelle au sein des actes de phantasia abolit du même coup la distinction de ce qui est réel et de ce qui est real22, sans pour autant rabattre le perçu sur le sensible. Le Wesen n'est à proprement parler ni sensible ni perçu, mais dynamique de phénoménalisation. Il ne qualifie pas une expérience de fusion pure avec le sensible, encore moins une expérience sans extériorité, mais l'ouverture à la manifestation ou la phénoménalisation d'un pré-être chose dont le format et le sens ne sont pas a priori déterminés ni normés. Cet état de base de la phénoménalisation est celui d'un clignotement ou d'une latence : quelque chose m'affecte, me retient plus ou moins, mais ne capture pas nécessairement son phénomène. Ainsi, lorsque je somnole et que je suis affecté par quelque chose, ma réaction et l'expérience de ce qui m'affecte peuvent être très fortes sans prendre immédiatement le format d'une identification perceptive. Tout le travail de refondation faisant de la phantasia le «terrain » de l'enquête phénoménologique vise restituer leur dignité à ces processus de phénoménalisation qui traversent l'expérience et dont les actes perceptifs ne sont que des moments.

19 Richir M, Phénoménologie en esquisses, Grenoble, Millon, 2000, p. 107.

20 Cf. Forestier F., La phénoménologie génétique.., op. cit., p. 37.

21 Tout aussi bien selon Richir, il n'est pas possible de thématiser séparément les actes de l'affectivité. Au niveau de la phantasia, il n'y a pas d'avantage à distinguer entre l'acte lui-même et l'affectivité $q$ u'entre le sentir et le senti. La phantasia doit être originairement pensée comme phantasia-affection. 
C.

Pour Richir, ces distinctions ne suffisent cependant pas à rendre compte de la nature des différents types d'actes, en particulier des spécificités des actes perceptifs.

II est nécessaire d'introduire un autre concept (également husserlien), celui d'actes positionnels et doxiques. Un acte doxique est un acte intentionnel d'une nature particulière, qui ne donne pas seulement son objet, mais le reconnait et le réidentifie dans son identité et sa singularité. La perception (pour Richir et Husserl) est doxique, car elle ne se borne pas à constater la présence d'un quelque chose ; elle le reconnait dans son être tel et le pose dans une certaine modalité (certitude, présomption, doute).

Pour autant, le doxique ne suffit pas non plus à caractériser le perceptif et caractérise aussi de nombreux actes imaginatifs. Pour Richir, il est impossible de distinguer imagination et perception par le caractère d'extériorité de la seconde, car celui-ci n'est pas donné à même l'acte. Il y a au contraire dans la perception quelque chose d'hallucinatoire, un moment de captation qui ne présente en tant que tel aucun caractère intrinsèquement distinctif attestant de la réalité de la chose perçu. La perception se distingue plutôt de l'imagination par son caractère doxique continu et cohérent, lié à une persistance instituée, qui relève d'un jeu de sédimentations, d'habitus. Elle constitue en d'autres termes un phénomène profondément humain, lié à l'institution d'un monde humain, qu'elle ne peut donc pas fonder puisque précisément, elle en procède.

Plus encore, la genèse des actes perceptifs et imaginatifs est incompréhensible si on ne prend pas en compte la dimension idéale qui les accompagne. Ce n'est pas la perception qui donne l'extériorité, mais bien plutôt l'horizon de l'extériorité qui appelle l'effectuation et la stabilisation d'actes perceptifs. Celle-ci présuppose un travail de stabilisation et de discipline symbolique, et cela d'autant plus que, pour Richir, l'horizon idéal de transcendance qui motive les actes perceptifs motive aussi des actes pseudo-perceptifs parasites, autrement dit, des hallucinations. L'extériorité suscite indistinctement perceptions et phantasmes, de telle façon que l'horizon de la vérité et de l'exactitude (de ce qui est à l'extérieur) est du même coup l'horizon du simulacre et de l'erreur, la teneur expérientielle ne suffisant seule à les discriminer.

Contrairement à la phénoménologie husserlienne, donc, la phénoménologie richirienne ne conduit à aucun sol apodictique, mais à un effort et un travail d'exploration et d'objectivation symboliquement cadrés. 


\section{Genèse et incarnation}

A.

D’une certaine façon, chez Husserl également, les actes perceptifs ne se déploient qu'à partir de synthèses et de constitution de formes noématiques. Il est difficile, au cours de ce processus, de discerner les proto-perceptions des proto-imaginations sans lesquelles la conscience intentionnelle ne pourrait poser ces formes noématiques. Mais pour Husserl, l'ensemble du processus est téléologiquement orienté vers la donation perceptive et l'objectivation : il ne saurait être question pour lui d'un enveloppement des actes perceptifs eux-mêmes par les actes de phantasia.

Chez Richir à l'inverse, la phénoménalisation relève de transpositions architectoniques (et non de relations de fondation), c'est-à-dire de l'instauration de modes de structuration ou de phénoménalisation dépendant génétiquement les uns des autres, mais sans que les caractères de la couche de base ne viennent garantir ceux de la couche transposée. L'eidétique husserlienne du monde de la vie ne vaut ainsi que du point de vue de la « temporalisation en présent ${ }^{23}$ » caractéristique des actes perceptifs et ne peut donc fournir de fondation ontologique à la connaissance humaine.

Il faut éviter ici de réifier les différentes "strates » que distingue Richir et leurs relations, nécessairement heuristiques et provisoires. Le mérite du concept de transposition architectonique est à la fois de rompre l'homogénéité structurelle des analyses de Husserl et de proposer lecture qui ne se limite plus aux actes individuels et à leur caractère. Pour Richir, il n'y a pas de sens à vouloir étudier des types d'actes isolés indépendamment de leurs inscriptions dans des configurations. Il faut bien sûr rester prudent lorsqu' on adopte cette approche " configurationnelle " et garder en tête que les registres architectoniques ne sont que des prismes et des grilles de lecture. Le passage d'une analyse en termes d'objet à une analyse en termes de structures d'ensemble implique aussi un changement de pratique dans la phénoménologie. Les registres architectoniques sont avant tout des concepts opératoires (au sens de Fink).

B.

Richir tente cependant de donner des descriptions plus précises des processus à l'œuvre au sein de la genèse en élaborant une phénoménologie de l'incarnation.

23 Cf. Forestier F., La phénoménologie génétique...r, op. cit., le chapitre « L'énigme de la temporalité », pp. 109-141. 
Dans ses Fragments phénoménologiques sur le Temps et l'Espace, il exploite pour ce faire le potentiel des concepts introduits par Husserl de Leiblichkeit, Leib et Leibkörper, qu'il relie aux concepts métaphysiques de chôra, de topos, de holon.

Richir distingue tout d'abord deux processus: 1) l'intégration de la motricité, de l'action du corps sur le monde, 2) la distinction du soi par rapport au monde. Ceux-ci ne sont pas superposés, mais coïncident dans la dimension du Leibkörper, qui appelle à rendre compte de deux dimensions entremêlées : l'altérité et l'extériorité. La distinction de soi comme objet de la sollicitude d'un autre est inséparable d'une intégration progressive du ressentir. Tout aussi bien, le sentiment de l'existence des autres est inséparable de celui de l'existence du monde.

La transcendance du monde extérieur ne se manifeste pas d'abord comme transcendance de choses mais comme sentiment d'une extériorité. Elle n'est pas fondée par la stabilité d'une expérience, puisque c'est au contraire le "sentiment » de la transcendance du monde qui donne un sens nouveau aux choses que je rencontre et réidentifie.

Encore une fois, cette ouverture est imaginaire et idéale avant d'être perceptive et cognitive. Selon Richir, le Leib de cette ouverture est d'abord l'effet du jeu contradictoire des tendances affectives et sensibles, en particulier du flottement intrinsèque de certaines sensations (en particulier liées à la vue, à l'ouïe) que rien ne rattache a priori au corps et qui se phénoménalisent d'abord en flottement libre avant de s'ancrer peu à peu dans des objets fixes et situés. En quelque sorte, je sais que le monde est extérieur avant qu'il ne me soit donné comme tel, et c'est par ce sentiment de l'extériorité que j'entre dans le monde des mots, des significations et des lois (avant même que j'en saisisse le sens) au sein duquel ma perception s'institue et s'affine.

Si ce Leib est amené à se centrer en un Körper, en d'autres termes si la centration égoïque est amenée à se déployer comme pouvoir d'agir corporel, Leib et Körper ne sont pas totalement superposables. Je n'habite pas parfaitement mon corps qui est " plus ou moins mien » et ne me confonds pas à lui comme capacité d'agir pure. La centration du Leib sur le Körper fait de celui-ci une réserve par rapport à l'actualité, une potentialisation, une virtualité (cela Husserl le dit déjà), mais également une opacité, un espace de latence et d'incertitudes. Le corps n'est pas pure puissance disponible : il est aussi un lieu de brouillage, un lieu de symbolisation, une énigme pour lui-même. L'incarnation ne constitue pas seulement l'espace extérieur qui est inséparable d'une "spatialisation intérieure " affective. L'ouverture aux choses n' est jamais seulement mondaine et jamais tout à fait mondaine : en toute rigueur, il n'y a d'ailleurs pas vraiment de monde pour Richir, sinon comme forme de cohésion mouvante, provisoire. L'extériorité n'est pas liée à ce qu'il y a ni 
au fait qu'il y a des choses : elle est l'attracteur invisible qui module le rapport à ces choses qui ne sont pas données une fois pour toutes dans leur extériorité mais toujours débordée par l'épaisseur virtuelle au sein de laquelle elles sont taillées.

C.

L'amorce de phénoménologie du rêve que Richir propose en discussion avec Merleau-Ponty ${ }^{24}$ est une bonne illustration de cette facticité mise en jeu par la genèse. Pour Merleau-Ponty, il faut comprendre le rêve à partir du corps. Le rêve effectue une épochè de la réalité du corps situé et l'expérience du rêve est celle d'un être au monde avec un corps sans poids. Le rêve n'est donc pas dénué d'incarnation, mais c'est une incarnation sans ancrage, un Leib sans Körper, porteur d'un être-au-monde sans poids.

Tout aussi bien, le rêve s'affranchit de l'horizon mondain de concordance. Le monde du rêve n'est pas Un. Certes, le rêve n'est pas une succession de lambeaux informes puisqu'il a bien sens, forme : mais cette forme est polymorphe, ce monder en incessante reprise, reconfiguration, recontextualisation, sans trame, sans traces ni prises, où jamais ne s'ouvre le distance et l'aménagement d'une position de repli réflexif au sein de cette distance. Ainsi, le rêve met en jeu une phénoménalisation anonyme, qui échappe à la maîtrise d'un ipse et qui permet la rencontre du monde comme d'un autre, d'un monde que je n'habite pas, que je ne connais pas, que rien ne me prépare à rencontrer, auquel rien ne permet de se stabiliser comme le monde.

L'intéressant est de constater qu'il n'y a finalement pas d'attestation permettant de distinguer clairement le rêve de la veille. La différence phénoménologique du rêve et de la veille n'est pas donnée par un contenu mais par un processus actif de redécouverte et réinstitution que le rêve ne permet pas. La veille concentrée, thétique, perceptive, n'est pas réceptivité d'une évidence mais effectuation toujours provisoire et partielle d'une réflexibilité transposée en acte et inscrite au sein d'une forme de vie qui en permet la temporalisation et la spatialisation.

\section{Conclusion}

Avec Richir, nous passons ainsi d'une phénoménologie de la donation à une phénoménologie de l'attestation active. L'enjeu n'en est plus de déterminer comment les sens, ou la perception, ou quelque autre instance, nous donneraient le

24 Richir M, Phénoménologie en esquisses, op. cit., p. 190. 
réel, ni même d'interroger les conditions de possibilités de notre rapport à lui, mais de comprendre notre activité formatrice comme seul lieu d'épreuve et d'attestation. Une telle perspective ne rompt pas tout à fait avec la pensée husserlienne de la genèse, reculant de l'ontologie prédonnée du monde de la vie aux actes de conscience qui les constituent. Seulement, le processus de sédimentation est ici multiple, mouvant, et toujours latent. La genèse est à la fois transcendantale et accidentelle, dans la mesure où elle n'est pas portée par la nécessité d'un déploiement téléologique, mais par une succession de transformations, de bouleversements et de stabilisations schématiques, dont l'origine est toujours aussi rituelle, technique, sociale, et symbolique.

On peut certes avec Husserl postuler une base naturelle à cette dynamique : le développement physiologique, comme héritage de la sélection naturelle, impulse d'emblée une direction de développement qui fait immédiatement et de façon quasi-indémêlable cercle avec les premières expériences sensibles dans un processus d'affinement sensoriel, perceptif et moteur. Contre Husserl cependant, il faut refuser d'assimiler ces tendances à une intentionnalité originaire " latente ", opératrice de «la validité du monde », une « vie transcendantale constituante ${ }^{25}$ ». La générativité est à la fois inséparable de la fixation symbolique et inséparable de l'activité configuratrice singulière qui la parachève.

S'il faut considérer, avec Etienne Bimbenet, qu'il y a eu, avec l'hominisation et l'humanisation, une véritable invention du réalisme et de l'attitude réaliste, dont les déterminants ont été à la fois biologiques et physiologiques, sociaux et linguistiques, celle-ci n'est pas plus le produit unilatéral d'une tendance vitale à la transcendance que celui d'une castration liée à l'apparition du langage où à l'environnement sociotechnique. Les deux voies de l'idéalité que distingue Bimbenet ${ }^{26}$ (celle de la " mort », de la rupture avec le monde, et celle de « l'élan », de la participation et de l'approfondissement) sont indémêlablement à l'œuvre au sein de cette Bildung phénoménologique qui ne cesse de former des objets et de discipliner des mondes. La phénoménologie nous apprend que la genèse n’est ni un processus spontané et définitif ni un flux hasardeux et fugace. C'est une activité toujours béante sur la réflexibilité qui la traverse.

25 Farges Julien, «Vie, science de la vie et monde de la vie : Sur le statut de la biologie chez le dernier Husserl. » in Bulletin d'analyse phénoménologique, Université de Liège, 2010, La nature vivante, 6 (2), pp. 42-72. http://popups.ulg.ac.be/1782-2041/index.php?id=362. halshs-01333672.

Bimbenet Etienne, L'invention du réalisme, Cerf, 2015, p. 150ss. 
Florian Forestier est conservateur à la Bibliothèque nationale de France et chercheur associé aux Archives Marc Richir de l'Université de Wuppertal. Il est l'auteur de plusieurs ouvrages dont La phénoménologie génétique de Marc Richir (Springer, Phaenomenologica, 2014), Le réel et le transcendantal (Jérôme Millon, Krisis, 2015), Le grain du sens (Zeta Books, 2016) et de nombreux articles. Il a également participé à la rédaction de rapports officiels, ainsi qu’à la conception du programme de recherches de la Stratégie nationale pour l'Autisme au sein des troubles du neuro-développement. 